\title{
On the Structure in Aqueous Superconcentrated Calcium Nitrate
}

\author{
R. Caminiti, A. Musinu, G. Paschina, G. Piccaluga, and G. Pinna \\ Istituto Chimico Policattedra, Università di Cagliari, Cagliari, Italy \\ Z. Naturforsch. 36a, 831-835 (1981); received March 9, 1981 \\ Liquid hydrous calcium nitrate of composition $\mathrm{Ca}\left(\mathrm{NO}_{3}\right)_{2} \cdot 3.5 \mathrm{H}_{2} \mathrm{O}$ was investigated by X-ray \\ diffraction. Experimental data were interpreted in terms of geometrical models suggested by the \\ structure of the crystalline hydrates $\mathrm{Ca}\left(\mathrm{NO}_{3}\right)_{2} \cdot 3 \mathrm{H}_{2} \mathrm{O}$ and $\mathrm{Ca}\left(\mathrm{NO}_{3}\right)_{2} \cdot 4 \mathrm{H}_{2} \mathrm{O}$, in which calcium \\ ions are coordinated to nine oxygen atoms, partly coming from water molecules, partly from \\ $\mathrm{NO}_{3}-$ ions.
}

\section{Introduction}

An overall view of $\mathrm{X}$-ray diffraction studies of ionic solutions shows that in a great range of concentrations $(0.5 \mathrm{M}<c<4-5 \mathrm{M})$, leaving apart cases in which complexation by the anions occurs, the structure of the solutions is mainly determined by hydration phenomena [1]. Thus, in the correlation functions calculated from experimental data, the only peaks structurally meaningful are those regarding interactions between the ions and first, and sometimes second, neighbour water molecules; ionion interactions, on the contrary, seem not to give rise to order phenomena of any great extent.

The "hydration structure" has proved to be quite stable. In fact, ion-water mean distances and mean coordination numbers (and sometimes even mean standard deviations of mean distances) seem to be almost insensitive to variations in concentration or changes of counter-ion, thus justifying the use of models with independent hydrated ions. In this regard it is worth noting that in several very concentrated solutions (like, for example, $\mathrm{LiCl}$ [2], $\mathrm{CaCl}_{2}[3]$ and $\mathrm{NiCl}_{2}[4]$ solutions) the coordination numbers found for both ions had the same value as those obtained in more dilute solutions, even though there was not enough water present to hydrate all the ions independently. It can be argued that the tendency to ion hydration will drive the water molecules towards positions in which they will satisfy at the same time the hydration needs of both cations and anions.

Very likely the first structural effect of reducing the water in the solution is thus a variation in the

Reprint requests to Dr. G. Piccaluga, Istituto Chimico Policattedra, Università di Cagliari, Via Ospedale 72, 09100 Cagliari/Italien. "conformation" of the water molecules neighbouring the central ion. This has been observed in a neutron diffraction study of $\mathrm{NiCl}_{2}$ solutions carried out by Neilson and Enderby [5], who used the technique of isotopic substitution; going from dilute to saturated solutions, a continuous variation in the tilt angle between the $\mathrm{Ni}-\mathrm{O}$ axis and the plane of the water molecule has been detected, with values going from $0^{\circ}$ to about $34-42^{\circ}$ degrees. However, as far as this aspect is concerned, $\mathrm{X}$-ray diffraction gives less direct information than neutron diffraction, so that not much can be said about this problem [1].

It would be really interesting to understand how, by further lowering the content of water in the system, the structural evolution goes on affecting even the "hydration structure" of the ions. Unfortunately, it is rather difficult to explore solutions whose composition more and more approaches the one of the corresponding crystal hydrates because of the limits given by salt solubilities at usual working conditions. In one of the few cases in which this has been done $\left(\mathrm{CaCl}_{2} \cdot 6 \mathrm{H}_{2} \mathrm{O}\right.$ fused salt [6]), the structure appeared to be fundamentally determined by the "memory" of the hydrated crystal structure. This forces the cations $\mathrm{Ca}^{2+}$ to a coordination with water molecules different from the one usually exhibited in solutions not so concentrated. In fact the $\mathrm{Ca}^{2+}$ ion has a coordination number of nine, which is typical of crystalline $\mathrm{CaCl}_{2} \cdot 6 \mathrm{H}_{2} \mathrm{O}$, whereas in solutions up to a concentration 4.5 molal coordination values very close to six have been proposed both by X-ray [3] and neutron [7] diffraction experiments.

In order to go more deeply into this matter, we started an investigation on an aqueous solution of calcium nitrate, since, from this salt, solutions of composition corresponding to its hydrated salts can be obtained at room temperature.

0340-4811 / $81 / 0800-0831 \$ 01.00 / 0$. - Please order a reprint rather than making your own copy. 
Not much information on the structure of $\mathrm{Ca}\left(\mathrm{NO}_{3}\right)_{2}$ solutions can be drawn from the literature. An X-ray diffraction study [8] on a solution $1 \mathrm{M}$ described the $\mathrm{Ca}^{2+}$ ion as having an octahedral coordination with water molecules, but this concentration is too far from the ones we are interested in. On the other hand, very concentrated solutions have been studied by Raman spectroscopy [9]. This technique showed some spectral evidence of $\mathrm{Ca}^{2+}-\mathrm{NO}_{3}{ }^{-}$contacts, but this does not give much information about the overall structure, as these contacts might come either from isolated ion pairs or from a "quasi lattice" spatial arrangement. Therefore, up to now, no complete description of the structure of superconcentrated aqueous calcium nitrate has been given.

\section{Experimental and Results}

The sample was prepared by warming gently tetrahydrated calcium nitrate, then bringing the melt thus obtained to $25{ }^{\circ} \mathrm{C}$. The sample concentration was obtained by passing a portion of the solution through a column with a cation exchanger and titrating the eluate with a standardized $\mathrm{NaOH}$ solution. The sample was found to have less water than the hydrated salt, so its stoichiometric composition was expressed by the formula $\mathrm{Ca}\left(\mathrm{NO}_{3}\right)_{2}$ $\cdot 3.5 \mathrm{H}_{2} \mathrm{O}$.

Diffraction data were collected with a $\theta-\theta \mathrm{X}$-ray diffractometer for liquids described elsewhere [3], using Mok $\alpha$ radiation $(\lambda=0.7107 \AA)$. Here, a divergent beam is diffracted on the horizontal surface of the sample and then monochromatized by reflection from a curved quartz crystal. Scattered intensities were collected at discrete points from $\theta=2^{\circ}$ to $\theta=68^{\circ}, 2 \theta$ being the scattering angle, corresponding to the range $0.62<s<16.44 \AA^{-1}$ $(s=4 \pi \sin \theta / \lambda)$. Steps in $\theta$ were of $0.1^{\circ}$ between $\theta=2^{\circ}$ and $\theta=20^{\circ}$ and $0.25^{\circ}$ between $\theta=20^{\circ}$ and $\theta=68^{\circ}$. The times required to accumulate 100000 counts at each point were recorded; the whole angular range was scanned twice.

The measured intensities were corrected for background, polarisation, absorption [10] and Compton modified radiation [2-4] and then scaled to absolute intensities using conventional methods [2-4]. Smoothing of the data by fourth differences was applied [11].
The structure function was then constructed according to

$$
i(s)=\left(I_{\mathrm{e}, \mathrm{u} .}-\sum_{i=1}^{m} x_{i} f_{i}{ }^{2}\right) /\left(\sum_{i=1}^{m} x_{i} f_{i}\right)^{2},
$$

where $f_{i}$ 's are the atomic scattering amplitudes corrected for anomalous dispersion, $x_{i}$ 's are the stoichiometric coefficients in a structural unit containing $m$ kinds of atoms and $I_{\text {e. u. }}$ is the intensity in electron units. The values of the $f_{i}$ 's used were those proposed by Hajdu [12] for the water molecules, by Cromer and Mann [13] for the $\mathrm{Ca}^{2+}$ and $\mathrm{O}^{-}$species and by Cromer and Waber [14] for the $\mathrm{O}$ and $\mathrm{N}$ neutral atoms.

The correlation function $G(r)$ was obtained from $i(s)$ by Fourier transformation according to

$$
\begin{aligned}
G(r)= & 1+\left(2 \pi^{2} \varrho_{0} r\right)^{-1} \\
& \cdot \int_{s_{\min }}^{s_{\max }} \operatorname{si}(s) M(s) \sin r s \mathrm{~d} s,
\end{aligned}
$$

where $r$ is the interatomic distance, $s_{\min }$ and $s_{\max }$ are the lower and the upper limit of the experimental data and $\varrho_{0}$ is the bulk density of stoichiometric units. $M(s)$ is a modification function of the form $e^{-b 8^{2}}$, which is a damping factor that minimizes the spurious peaks in $G(r)$ due to the truncation of the integral at $s_{\max }$; the value of $b$ used was 0.005 .

The si $(s)$ and $G(r)$ functions from experiments are shown in Figs. 1 and 2 respectively (dots). The

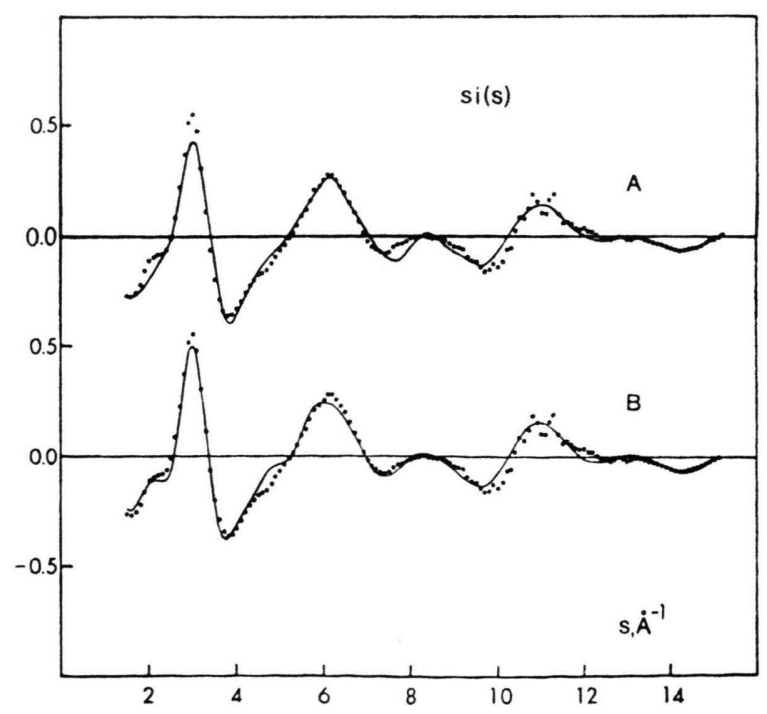

Fig. 1. Experimental (...) and model (-) structure functions are given for the two models used. 


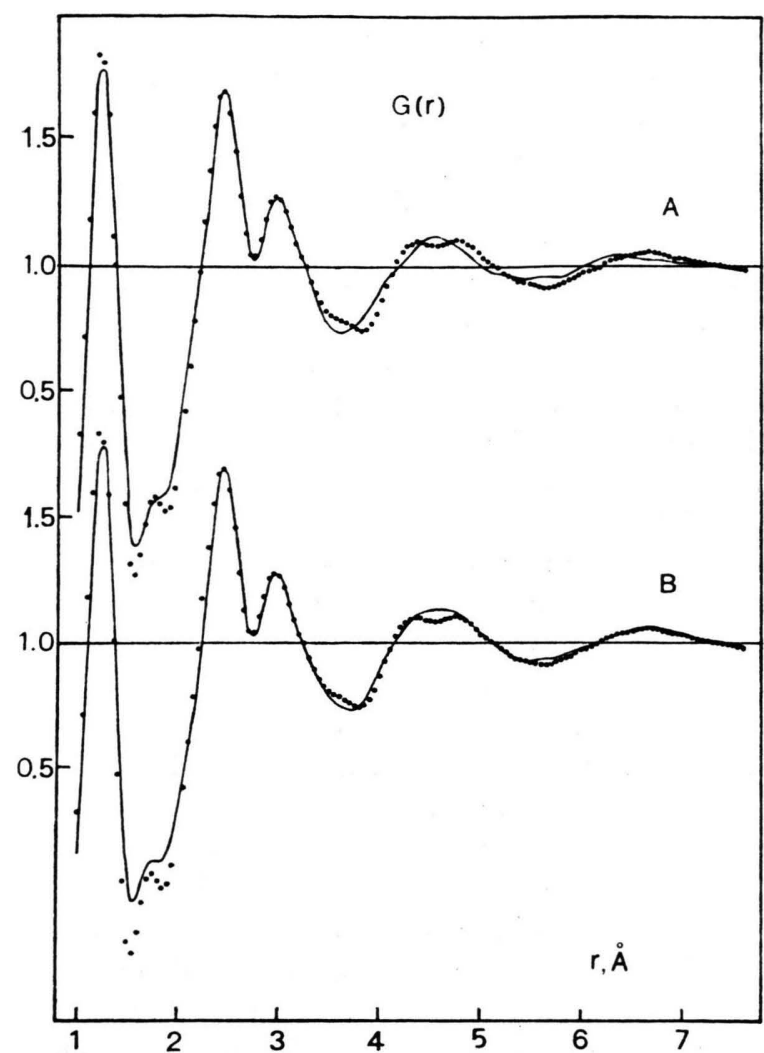

Fig. 2. Experimental (...) and model (-) correlation functions are given for the two models used.

si $(s)$ function was corrected for low-frequency contributions leading to spurious peaks below about $1 \AA$ in the $G(r)$ function [2-4].

\section{Discussion and Conclusion}

The experimental correlation function shows three main peaks at about $1.25 \AA, 2.45 \AA$ and $2.95 \AA$, and two broad bands at 4-5 $\AA$ and $6-7 \AA$, respectively (besides these peaks, another one can be seen at about $1.70 \AA$, but it must be considered spurious, as no real distance of this value can exist in the system).

The short range order suggested by these peaks is consistent with that existing in the starting solid hydrate, but also with that of another crystalline hydrate of composition close to our sample, which is tri-hydrated calcium nitrate. As we shall soon see better, in the two hydrates [15-16] distances corresponding to the ones mentioned are present, as coming respectively from $\mathrm{N}-\mathrm{O}$ interactions inside the nitrate ions, from interactions $\mathrm{Ca}^{2+-} \mathrm{O}$ (with the oxygen either of the nitrate ion or of the water) and from interactions of $\mathrm{O}-\mathrm{O}$ and $\mathrm{N}-\mathrm{O}$ type. Then, considering the concentration of the sample, the structures of the crystalline salts (tri- and tetra-hydrated) were regarded as reasonable models for the sample under investigation.

Model $\mathrm{A}: \mathrm{Ca}\left(\mathrm{NO}_{3}\right)_{2} \cdot 4 \mathrm{H}_{2} \mathrm{O}$

The structure of tetra-hydrated calcium nitrate [16] is in the form of centrosymmetric dimeric units $\left[\mathrm{Ca}\left(\mathrm{NO}_{3}\right)_{2} \cdot 4 \mathrm{H}_{2} \mathrm{O}\right]_{2}$. The calcium atoms are bounded through bridging oxygen atoms from nitrate ions. Each calcium atom is surrounded by 9 oxygens, five of which come from nitrate ions and four from water molecules. The coordination polyhedron resembles a triangular base prism whose lateral sides form square-base pyramids. Crystalline structure cohesion is achieved through bonds of different type between one centrosymmetric unit and eight identical units surrounding it.

Model $\mathrm{B}: \mathrm{Ca}\left(\mathrm{NO}_{3}\right)_{2} \cdot 3 \mathrm{H}_{2} \mathrm{O}$

According to Leclaire [15], the monoclinic crystals are formed by cyclic tetrameric units $\left[\mathrm{Ca}\left(\mathrm{NO}_{3}\right)_{2}\right.$ $\left.\cdot 3 \mathrm{H}_{2} \mathrm{O}\right]_{4}$. The calcium atoms in the rings are approximately set in a plane of (001) type and linked by nitrate ions. The four calcium atoms are coordinated to nine oxygen atoms, six of which come from nitrate ions and three from water molecules. In the coordination polyhedra thus obtained eight vertices define an Archimedean distorted antiprism and the ninth forms a pyramid with an antiprism quadrangular side. The crystal structure is made up of layers parallel to the plane (001). Inside the layers the tetramers are $\mathrm{H}$-bonded to each other, just as the layers themselves are H-bonded to each other.

A detailed examination of all $\mathrm{Ca}-\mathrm{O}$ distances shows that, in both models, the bonds between calcium and oxygen from water molecules are shorter than the ones between calcium and oxygen from nitrate ions, mean distances being $2.410 \AA$ and $2.555 \AA$ respectively in the tri-hydrate and $2.451 \AA$ and $2.575 \AA$ respectively in the tetra-hydrate. Moreover, the nine oxygen atoms are, in both models, at different distances from the central atom; besides that, in the tri-hydrated from each $\mathrm{Ca}$ atom has a (although slightly) different spectrum of $\mathrm{Ca}-\mathrm{O}$ distances.

Going a bit further, we formulated the hypothesis that the units present in the melted salt are 
the same (be they dimers or tetramers) as the ones forming the hydrated salts $\mathrm{A}$ and $\mathrm{B}$, while the $\mathrm{H}$ bonds, which in the solid link together such units, are here broken. Discrete distances must therefore exist inside the dimeric or tetrameric units, but these should be completely random the one to the other.

From these models synthetic structure functions were calculated and refined against experimental data. As in previous papers [2-4], the contribution from the discrete interactions is accounted for by using the Debye formula modified so that a Gaussian-type distribution of interatomic distances can be introduced. As discrete distances inside the assumed units go beyond the position $(6-7 \AA)$ of the last meaningful peak of experimental $G(r)$, we decided to omit in the synthetic si $(s)$ functions the terms describing a continuous distribution of distances ("continuum"). It followed that the initial range of the structure function $\left(s<1.0 \AA^{-1}\right)$ had to be left out of the refinement procedure.

In the structure thus far described, the atoms are so linked together that a given pair distance cannot be modified without altering the whole distance spectrum. Therefore mean positions of atoms in dimeric or tetrameric units were assumed as being exactly the ones given by Leclaire [15] and Leclaire and Monet [16]; none of the inside-unit distances were then considered as independent parameters. The only independent parameters used were the mean square deviations of the interatomic distances, which account for the disorder inside the structural units caused by the melting process. However, to each distance falling within a present range (for example, $1.0-1.8 \AA, 1.8-2.3 \AA, 2.3-$ $2.5 \AA$ and so on) the same value of mean square deviation was assigned, so that the number of independent parameters came out much less than the number of pair distances existing in the models.

Besides that, as the amount of water exceeds that of tri-hydrated salt, interactions between free water molecules were added in the case of model B; for these interactions also the interatomic distance was refined.

The calculations proved that both models are consistent with the experimental data. This is shown in Fig. 1, where synthetic structure functions of the two models are compared with experimental data. In order to have a comparison between correlation functions, the theoretical $\mathrm{si}(s)$ 's were connected to the experimental data at $s=1.5 \AA^{-1}$ and then Fourier transformed. In Fig. 2 the correlation functions thus obtained are plotted against the ones from the experimental data. In model A the agreement is worse at low $s$ values in the structure function and, conversely, at high $r$ values in the correlation function; the differences, however, are not such as to exclude the correctness of the model. The comparison of the experimental and theoretical radial curves supplies a clear confirmation of the spurious nature of the small peak at $1.70 \AA$; in fact, it is observable also in the $G(r)$ 's calculated from the synthetic structure functions, although no discrete interaction with this characteristic mean distance exists in the models. Final values of refined parameters are given in Table 1 for both models. One could find it strange that both crystalline models used are consistent with the experimental data. The difficulty in discriminating between the models chosen can be explained by considering that in both models the interatomic distances within the units taken are distributed in an almost continuous way. From it come the broadened peaks observed in the radial functions, as well as the type of short and medium-range distribution which is rather alike in the two models.

Keeping this in mind, some elements seem to support the existence of an order derived from crystalline solid structures. First, the agreement between models and experiments was reached without refining the interatomic distances which were taken from crystalline models (except the not important water-water distance in the model B). Second, the final picture of the independent param-

Table 1. The final values of the independent parameters, with their standard errors in parentheses, are given for the two models used.

\begin{tabular}{lll}
\hline & Model A & Model B \\
\hline$\sigma_{1.0 \AA \leqq r<1.8 \AA}$ & $0.02(3)$ & $0.016(6)$ \\
$\sigma 1.8 \AA \leqq r<2.3 \AA$ & $0.14(2)$ & $0.13(1)$ \\
$\sigma 2.3 \AA \leqq r<2.5 \AA$ & $0.124(6)$ & $0.108(6)$ \\
$\sigma 2.5 \AA \leqq r<2.7 \AA$ & $0.22(1)$ & $0.170(9)$ \\
$\sigma 2.7 \AA \leqq r<3.0 \AA$ & $0.11(1)$ & $0.113(8)$ \\
$\sigma 3.0 \AA \leqq r<3.5 \AA$ & $0.16(1)$ & $0.213(9)$ \\
$\sigma 3.5 \AA \leqq r<4.0 \AA$ & $0.31(4)$ & $0.37(3)$ \\
$\sigma_{4} .0 \AA \leqq r<4.5 \AA$ & $0.29(1)$ & $0.265(9)$ \\
$\sigma_{4} .5 \AA \leqq r<5.0 \AA$ & $0.20(2)$ & $0.275(9)$ \\
$\sigma 5.0 \AA \leqq r<6.0 \AA$ & $0.46(5)$ & $0.48(1)$ \\
$\sigma_{6} .0 \AA \leqq r<7.0 \AA$ & $0.15(4)$ & $0.36(1)$ \\
$\sigma_{r} \geqq 7.0 \AA$ & $0.31(8)$ & $0.66(5)$ \\
$r_{\mathrm{H}_{2} \mathrm{O}-\mathrm{H}_{2} \mathrm{O}}$ & - & $2.917(5)$ \\
\hline
\end{tabular}


eters is quite reasonable; in fact, the mean square deviation values tend to increase with the interatomic distance, as expected because of the loss of positional correlation at increasing distances (model $\mathrm{B}$ has the more regular pattern). Finally, the structure factor, even without terms describing the "continuum", has been satisfactorily reproduced in the entire $s$ range used in the fitting procedure; that should mean that, not only nearest neighbour distances, but even the medium range order of the models used, are consistent with the experimental data.

The consistency between models and experimental data is, of course, only a necessary condition for the models to be judged valid. Possibly a "hydration type" model with well chosen parameters could reach as good a fit with the experimental structure functions as the "crystal type" models considered in the present paper. Our approach,

[1] R. Caminiti, G. Licheri, G. Piccaluga, G. Pinna, and M. Magini, Rev. Inorg. Chem. 1, 333 (1979).

[2] A. H. Narten, F. Vaslow, and H. A. Levy, J. Chem. Phys. 58, 5017 (1973).

[3] G. Licheri, G. Piccaluga, and G. Pinna, J. Chem. Phys. 64, 2437 (1976).

[4] R. Caminiti, G. Licheri, G. Piccaluga, and G. Pinna, Faraday Discuss. Chem. Soc. 66, 62 (1977).

[5] G. W. Neilson and J. E. Enderby, J. Phys. C. 11, L 625 (1978).

[6] G. Licheri, G. Piccaluga, and G. Pinna, J. Amer. Chem. Soc. 101, 5438 (1979).

[7] S. Cummings, J. E. Enderby, and R. A. Howe, J. Phys. C. 13, 1 (1980). however, together with the one already mentioned for melted $\mathrm{CaCl}_{2} \cdot 6 \mathrm{H}_{2} \mathrm{O}$, raises new questions in the field of structural investigations on aqueous solutions of electrolytes. It is in fact plain that the results obtained in the case of mean concentration solutions cannot be extrapolated, not even as a first approximation, to limit-concentration solutions, as those examined here.

Therefore, new data and maybe more powerful methods are needed to get information about the transition from the "hydration structures" to others that we can name "solid type structures".

\section{Acknowledgements}

This work was partially supported by the Consiglio Nazionale delle Ricerche. Calculations were carried out in part at the Centro di Calcolo dell'Università di Cagliari and in part at the Centro di Calcolo Interfacoltà dell'Università di Roma.

[8] W. Bol, G. J. A. Gerrits, and C. L. Van Panthaleon Van Eck, J. Appl. Cryst. 3, 486 (1970).

[9] B. Balshaw and S. I. Smedley, J. Phys. Chem. 79, 1323 (1975) and references therein quoted.

[10] M. E. Milberg, J. Appl. Phys. 29, 64 (1958).

[11] C. Lanczos, Applied Analysis, Prentice Hall, Englewood Cliffs (1956).

[12] F. Hajdu, Acta Cryst. A 28, 250 (1972).

[13] D. T. Cromer and J. B. Mann, Acta Cryst. A 24, 321 (1968).

[14] D. T. Cromer and J. T. Waber, Acta Cryst. 18, 104 (1965).

[15] A. Leclaire, Acta Cryst. B32, 235 (1976).

[16] A. Leclaire and J. C. Monier, C. R. Acad. Sci. Paris 25, 1555 (1970). 